\title{
Das Gräberfeld am Windmühlenberge bei Klein- Quenstedt, Kr. Halberstadt
}

\author{
Von Hugo Mötefindt
}

Eine durch ihren Siedlungsreichtum für die Vorgeschichtsforschung besonders wichtige Gegend ist die Umgebung Halberstadts. Hier ist der Bernburger Typus heimisch, ${ }^{1}$ ) hier ist die Kugelamphore nebst begleitenden Formen zahlreich vertreten, ${ }^{2}$ ) hier ist die Bandkeramik, aus dem südöstlichen Donaulande stammend, ebenfalls stark verbreitet, ${ }^{3}$ ) ebenso die Mischstile des Rössener Typus ${ }^{4}$ ) und der schnitt-, furchenstich- und schnurverzierten Keramik ${ }^{5}$ ) sowie die sporadisch weitverbreiteten kupferzeitlichen Zonenbecher. ${ }^{6}$ ) Ganz besonders auffällig jedoch tritt diese starke Besiedlung in der ältesten Bronzezeit ${ }^{\circ}$ ) hervor: die Aunjetitzer Keramik ist

1) Grabfunde: Lausehügel zwischen Mahndorf und Derenburg: 26 Gefässe im Museum Wernigerode. - Kloster Groningen und Sargstedt im Museum Halberstadt. Fine grosse Ansiedlung auf dem Bocksherge bei Derenburg; unter den Scherben auch mehrere Bruchstücke von Kragenflaschen (Museum Halberstadt und Wernigerode). Nach A. Crötze ("Cbersicht über die Vor- und Frühgeschichte Thüringens" in GötzeHöfer-Zschiesche, Die vor- und frühgeschichtlichen Altertümer 'Thüringens S. XXII) begleiten diesen 'lypus durch 'Thüringen und durch Brandenburur kleine vierkantige Beile aus Wiedaer Schiefer. Dieses Beil ist in der Halberstädter Gegend heimisch: hier stebt der Schiefer an und ist ausserordentlich viel verarbeitet. Allein in Gross-Quenstedt sind 43 Exemplare gesammelt, auf den Spiegelsbergen 38. (Bärthold, Führer durch die rorgeschichtliche Abteilung des städtischen Museums in Halberstadt. 1911. S. 22.)

2) P. Z. II, 347ft. - Ausserdem besonders schöne Exemplare aus Sargstedt in der Sammlung Germann-Sargstedt (Publikation wird vorbereitet). Fin Scherben von Mahndorf und ein ${ }^{2}$ weitmundiger Topf ${ }^{*}$ ron Zilly, Kr. Halberstadt, im Museum Wernigerode.

3) Viele Einzelfunde aus Wohn- und Herdgruben im Museum Halberstadt und in der Sammlung Germann-Sargstedt. Vielleicht gehört hierher auch ein Grabfund von Harsleben, Kr. Halberstadt (Museum Halberstadt), den ich demnächst zu veröffentlichen beabsichtige. Zwei Gefässe von Derenburg, Kr. Halberstadt, und Scherben von Sargstedt im Museum Wernigerode.

4) Mahndorf, ein kesselförmiger Becher. Altgatersleben, ein Becher wie P. Z. I, Taf. XXXVIII, 3. Beide im Iuseum Wernigerode.

5) Fine Amphore und ein Becher im Kloster (ironingen, eine Scherbe von Sargstedt im Museum Halberstadt. Haus Neindorf: 2 Amphoren. Halberstadt: 1 Becher. Dardesheim: 1 Amphore. Museum Wernigerode.

6) Grabfund von Schwanebeck im Museum Halberstadt. Armschutzplatte von Zilly, Kr. Halberstadt, im Nuseum Wernigerode.

7) Vgl. A. Götze, Bronzezeitliche Hockergräber bei Halberstadt. P. Z. II, 60ff. Höfer, Frühbronzezeitlicher Grabfund von Derenburg, Kr. Halberstadt, Jahresschrift für die Vorgeschichte der sächsisch-thüringischen I.änder IX. 1910, S. 61.

- Derselbe, Depotfund von Ziegelsberge bei Halberstadt. Ebendort V, 19)6, S.94ff.

- Derselbe, Funde ron Derenburg. Fbendort V, 1906, S 92 ff. 
hier so zahlreich in Gräbern gefunden, dass man sie nach ihrem stärksten Auftreten in Deutschland nach dem Vorgange Höfers ${ }^{1}$ ) mit gutem Recht Halberstädter Typus nennen könnte.

Besonders reich an Schätzen dieses Typus ist das städtische Museum in Halberstadt. Von seinen Funden war jedoch bisher noch nichts veröffentlicht. Durch die Freundlichkeit des Herrn Oberprediger Bärthold bin

$$
\begin{aligned}
& \text { Sagzplan der Gräter am Wirdmïhlenberge } \\
& \text { ber Klin - Guensizdt. } \\
& \begin{array}{lll}
\mathrm{s} & 10 & 15 \mathrm{~m} \\
\hline
\end{array}
\end{aligned}
$$

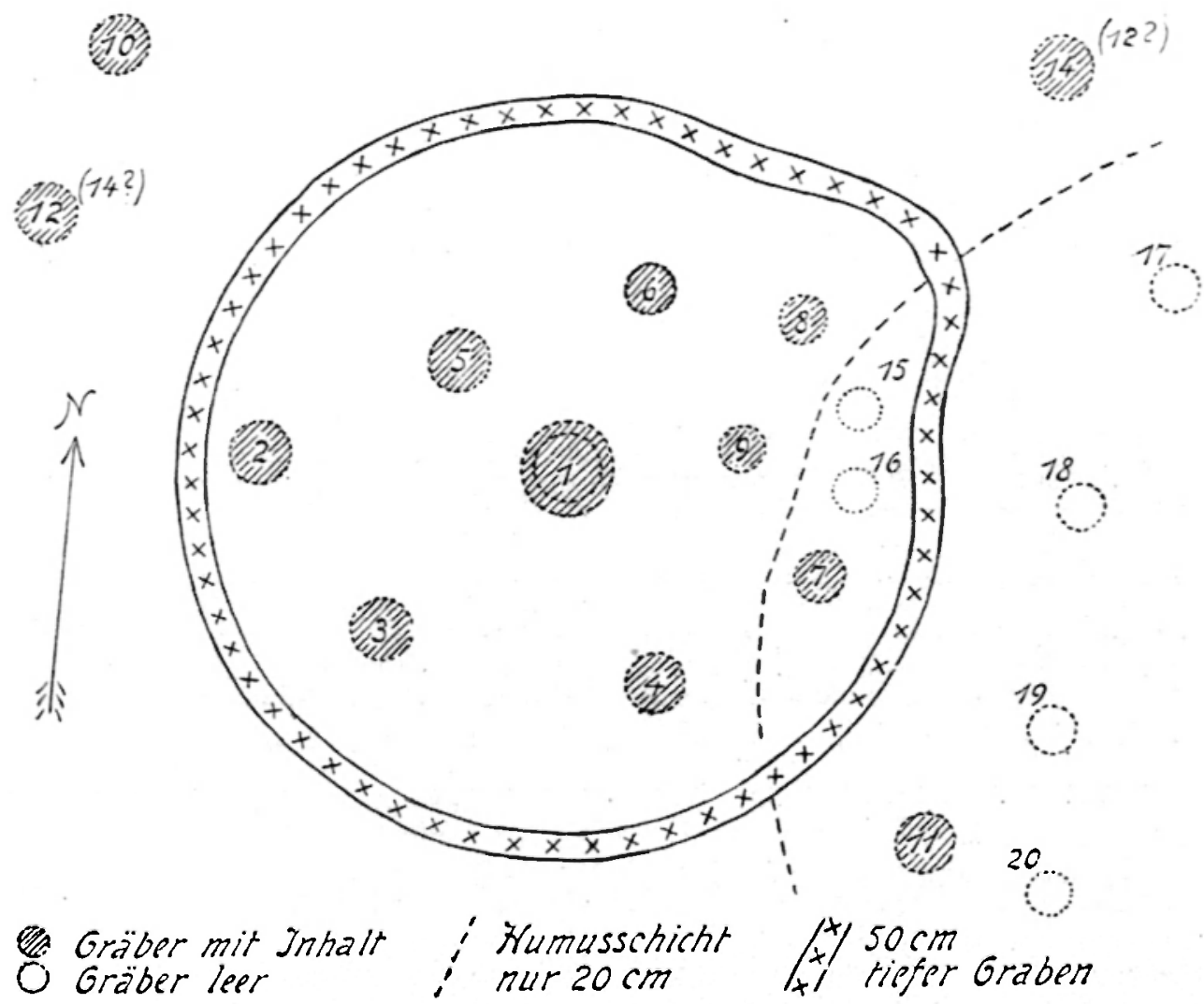

Abb. 1 .

ich in der Lage, jetzt all diese Aunjetitzer Funde veröffentlichen zu können. Den Anfang mache ich mit einem Gräberfeld. das neben dem Aunjetitzer Typus auch Funde anderer Zeiten barg, das aber besonders durch ein Grab mit zwei übereinanderliegenden Bestattungen von jenem Typus ausserordentlich interessant ist.

Am Windmühlenberge bei Klein-Quenstedt, $\mathrm{Kr}$. Halberstadt, wurde in

1) Jahresschrift $1 \mathrm{X}, 1910$, S. 93. 
der Halberstädter städtischen Kiesgrube (Lageplan Abb. 1) im August 1903 dieses Gräberfeld aufgedeckt. Die Ausgrabung leitete der Apotheker Maak. Durch die Vorbereitung meiner Veröffentlichung von Kugelamphoren aus dem Halberstädter Museum wurde ich darauf geführt, auch die Gefässe aus diesem Gräberfelde zu bearbeiten.

Es standen mir weiter keine Notizen zur Verfügung als ein Plan des Gräberfeldes (Abb. 1), eine Zeichnung des Doppelgrabes (Abb. 2), beide im Museum befindlich und von der Hand Maaks herrührend. Durch Herrn Mittelschullehrer Hemprich erhielt ich eine Reihe von Photographien, die Maak auf Karton aufgezogen hatte, und die von seiner Hand einige Bemerkungen trugen.

Vermutungen, dass dieser oder jener noch Notizen über die einzelnen Gräber besitzen könne, erwiesen sich bei näherer Erkundigung als trüge-

$$
\text { Trofil des Grabis } 1
$$

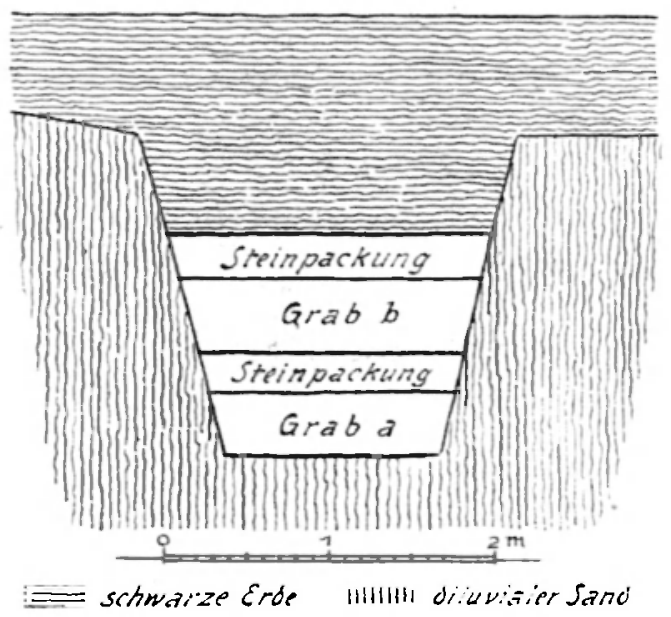

Albb. 2. Kl. Quenstedt, Windmühlenberg. risch. Ein Besuch der Kiesgrube war gleichfalls vergeblich, da die Arbeiter inzwischen of $t$ gewechselt hatten. So blieb mir weiter nichts übrig, als die Funde selbst und die bei ihnen liegenden Etiketten, die wenigstens teilweise neben der Inventarnummer auch die Nummer der Gräber angaben. Durch freundliche Unterstützung des Herm Oberprediger Bärthold, dem ich auch an dieser Stelle meinen herzlichsten Dank dafür ausspreche, ist es mir mit vieler Mühe gelungen, den Inhalt einiger Gräber zusammenzustellen.

Grab 1. Von diesem Grabe befindet sich im Museum eine von Maak gezeichnete Skizze; eine andere, gleichfalls von Maak herrührende

Skizze dieses Grabes habe ich durch Herrn Mittelschullehrer Hemprich erhalten. Beide Skizzen stimmen im wesentlichen überein; nur auf der letzteren sind die Gefässe in anderer Zusammenstellung eingetragen; ich glaube aber, dass die im Museum hängende Skizze, weil sie allein von Maaks Hand herrührende ausführlichere Angaben über die Form der Gefässe enthält, die allein richtige ist. Nach beiden Skizzen hatte das Grab folgendes Profil (Abb. 2): Unter der oberen, etwa $50 \mathrm{~cm}$ starken Schicht schwarzer Erde ergab sich eine in den diluvialen Sand eingelassene Grube; Tiefe unter dem Erdboden bis zur Sohle etwa 2,50 m, Breite unter.der Humusschicht 2,30 m, Breite an der tiefsten Stelle 1,20 m. Ob diese Grube aber rund oder viereckig war, ist nicht angegeben; das letztere ist jedoch wohl wahrscheinlicher. Unter der oberen Schicht schwarzer Erde folgte in der Grube eine zweite von etwa $80 \mathrm{~cm}$ Stärke, aus feiner schwarzer Erde bestehend. Hierunter lag eine $20 \mathrm{~cm}$ dicke Steinpackung, darunter als obere Bestattung ein Skelett ${ }^{1}$ ) mit zwei Gefässen.

Von diesen Gefässen ist das eine ein kleiner Napf (Inv. 294), Höhe $6 \mathrm{~cm}$, Mündungsdurchmesser $8,5 \mathrm{~cm}$, von einer Form, die sonst mit Verzierung im

1) $\mathrm{Ob}$ gestrecktes Skelett oder liegender Hocker, ist nicht angegeben. 
Bernburger Typus häufiger vorkommt ${ }^{1}$ ). Ein gleiches Gefäss wurde zusammen mit einem Aunjetitzer Topf bei Gross-Quenstedt, Kr. Halberstadt, gefunden (Museum Halberstadt).

Das zweite Gefäss soll eine Tasse sein; dieses Gefäss liess sich leider nicht menr ermitteln. Auf Hemprichs Tafeln sind vier Gefässe als „Beigabegefässe des grossen Grabes" zusammengestellt. Darnach müsste es sich um die Inventarnummer 278 handeln; nach meinen Ermittlungen stammt aber dieses Gefäss, eine etwas abgeschwächte Form des Aunjetitzer Bechers, aus Grab 7 (siehe dort). Auf einer anderen von den Tafeln steht folgende von Maaks Hand herrührende Bemerkung: „Schalenurnen aus den flachen Gräbern, die Tassen aus dem tiefen Grabe." Darnach könnte es sich nur um eine von den Tassen J.-N. 316 oder 317 handeln, von denen es sich nicht mehr feststellen liess, in welchem Grabe sie gefunden. Sicheres lässt sich über dieses Gefäss nicht mehr aussagen.

Unter dieser ersten Bestattung folgte wieder eine etwa $20 \mathrm{~cm}$ starke Steinpackung, und darunter eine zweite Bestattung, ein Skelett mit folgenden Beigaben: zwei Gefässe, ein Feuersteinmesser, ein Feuersteinschaber, ein Knochenpfriemen. Die Beigabegefässe sind folgende: a) ein typischer Henkeltopf mit vier Füssen $^{2}$ ) (J.-N. 312, Abb. 4), über dem Umbruch verziert mit drei eingeritzten Linien;
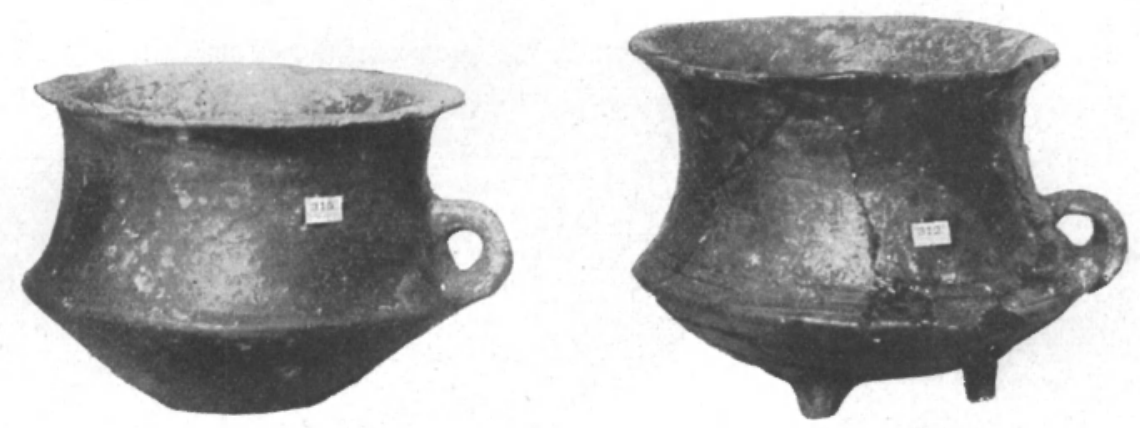

Abb. 3-4. Becher von Kl. Quenstedt, Windmühlenberg. Etwa 1/s nat. Gr. Museum Halberstadt.

alle drei Linien laufen unter dem Henkel hindurch. Die Höhe des Gefässes ist $11,5 \mathrm{~cm}$, der Mündungsdurchmesser $14,5 \mathrm{~cm}$, die Höhe jedes der vier Füsse etwa $2 \mathrm{~cm}$. b) Das zweite Gefäss ist gleichfalls ein Henkeltopf typischer Form (Abb. 3, J.-N. 315), Höhe $10 \mathrm{~cm}$, Mündungsdurchmesser $15 \mathrm{~cm}$. Über dem scharfen Umbruch ist eine schwache eingeritzte Linie sichtbar, und zwar tritt sie auf der Photographie deutlicher hervor als auf dem Original. Die anderen Beigaben dieser unteren Bestattung sind ein Knochenpfriemen mit sehr scharfer Spitze von $6 \mathrm{~cm}$ Länge, ein Feuersteinschaber von $7 \mathrm{~cm}$ Länge und ein Feuersteinmesser von $4,5 \mathrm{~cm}$ Länge.

Dieses Grab ist für die Chronologie der Halberstädter Formen äusserst wichtig. Es zeigt uns, dass die als lokale Eigentümlichkeit anzusehende Form von Aunjetitzer Bechern mit Füssen unter die ältesten Formen zu rechnen sind. Es ist nur zu bedauern, dass sich über das zweite Gefäss aus der oberen Fundschicht nichts ermitteln lässt.

1) Z. B. Osterode am Fallstein: M. Wernigerode. Jahresschr. VII, S. 27, Taf. VIII, 6.

2) Parallelen zu diesem Gefäss: Klein-Quenstedt (J.-Nr. 311 und 613, J.-Nr. 311 sogar mit fünf Füssen); im Museum Halberstadt, ebenfalls von Klein-Quenstedt ein Becher der im Museum Halberstadt ausgestellten Sammlung Ahlfeldt. Nr. 10 ein Becher mit der Bezeichnung Halberstadt im Museum Halle. - Publikation wird vorbereitet. 
Nun mag folgen, was sich aus meinen Nachforschungen über die anderen Gräber ermitteln liess: die Gräber Nr. 2 bis 13 waren zum grössten Teil Flachgräber mit (wahrscheinlich gestreckten) Skeletten; die Skelette lagen in der blossen Erde, neben ihnen standen die Gefässe. Mehrere Skelette hatten jedoch keine Beigaben; vielleicht sind dies die Gräber Nr. 3, 6 und 10. Torger hatte am meisten Interesse für die Skelette, so kam das andere zu kurz.

Grab 2. Ats diesem Grabe stammt vielleicht die Urne Abb. 5 (Inv. 262). Jedoch ist nach einer Mitteilung des Herrn Oberprediger Bärthold es wahrscheinlicher, dass sie in Grab 4 gefunden wurde. Unter den von Maak herrührenden Photographien steht angegeben Grab 5. Es handelt sich um einen Rauhtopf der La-Tene-Zeit, ${ }^{1}$ ) der mit Leichenbrand angefüllt war. Höhe $27 \mathrm{~cm}$, Mündungsdurchmesser $18 \mathrm{~cm}$.

$\mathrm{Gra} \mathrm{b}$ 3. Inhalt unbekannt.

Grab 4. In diesem Grabe befanden sich drei Gefässe, „eine grössere Urne und zwei einhenklige Schalen“. Mit der grösseren Urne könnte nur das

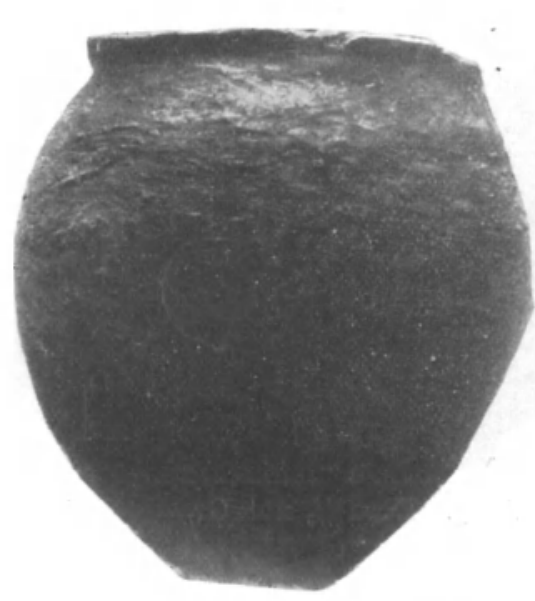

Abb. 5. Kl. Quenstedt, Windmühlenberg. $1 / 5$ nat. Gr. Inseum Halberstadt.

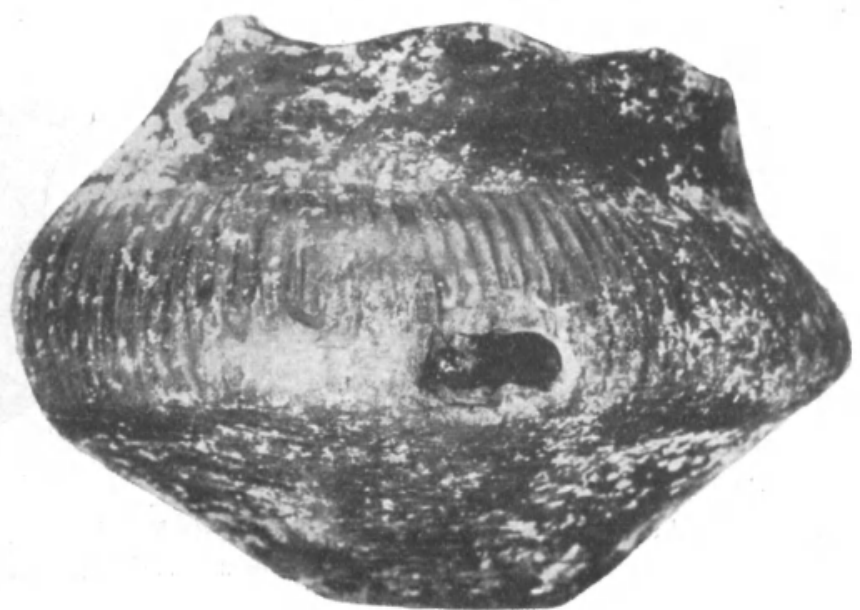

Abb. (6. Kl. Quenstedt, Windmühlenberg. $1 / 3$ nat. Gr. - Museum Halberstadt

Gefäss Abb. 5 (Inv. 262) gemeint sein (siehe Grab 2); über die anderen Gefässe ist überhaupt nichts zu ermitteln.

Grab5. Nach einer Notiz von Maaks Hand steht unter der Photographie des Gefässes Abb. 5 (Inv. 262) Grab 5.

G r a b 6. Inhalt unbekannt.

Grab 7. Aus diesem Grabe stammt ein Henkeltopf mit bedeutend in die Höhe gezogenem Umbruch (Inv. 278). Höhe $12 \mathrm{~cm}$, Mündungsdurchmesser $12 \mathrm{~cm}$. $\mathrm{Grab} 8$ lieferte einen geschweiften Becher (Inv. 282). Höhe $14 \mathrm{~cm}$, Mündungsdurchmesser $15 \mathrm{~cm}$. Eine Zeitbestimmung dieses Bechers ist mir ungewiss. Man könnte ihn wie den ihm ähnlichen Becher von Eisleben ${ }^{2}$ ), als Glockenbecher auffassen; seiner Form nach lässt er sich mit den verzierten Glocken-

1) Ein ähnliches Gefäss aus dem La-Tenezeitlichen Urnenfriedhof von Walternienburg, (Tulidenhügel), Kr. Jerichow I. Jahresschrift VIII. Taf. XXII 64/68. - Die Jahresschrift VI S. 89 ff. publizierten Funde vom Bernburg-Walternienburger Typus sind im Prov.-Nuseum Halle auch unter der Bezeichnung "Urnenfriedhof Walternienburg" (sic!) ausgestellt, trotzdem diese Funde doch aus skelettgräbern ohne Steinschutz stammen.

2) Jahresschrift VIII, Taf. III, 21. 
bechern von Bernburg, Welbsleben und Rottleben ${ }^{1}$ ) vergleichen; auch die Masse würden hier am besten herpassen. Andererseits zeigt er jedoch wieder Ähnlichkeiten mit gewissen Formen der Hallstattzeit.

Grab 9. Aus diesem Grabe stammen zwei Gefässe. Das eine (Inv. 313), ist ein Aunjetitzer Topf mit in die Höhe gezogenem Umbruch; der Henkel sitzt mit seinem unteren Ansatz unter dem Umbruch. Höhe $11 \mathrm{~cm}$, Mündungsdurchmesser $11,5 \mathrm{~cm}$. Das zweite Gefäss ist ein kleiner, aus rotem Ton gefertigter Becher ohne Henkel (Inv. 314), der mit acht eingeritzten Linien verziert ist, eine neue Form der Beigefässe vom Aunjetitzer Typus. Höhe 7,5 cm, Mündungsdurchmesser $9 \mathrm{~cm}$.

G r a b 10. Inhalt unbekannt.

Gra b 11. Aus Grab 11 stammt das Gefäss Abb. 6 (Inv. 319), ein Bruch. stück einer Lausitzer Amphore mit Hohlkehlen. Höhe $18 \mathrm{~cm}$, Mündungsdurchmesser nicht angebbar, da das Gefäss nur halb erhalten ist. Der Hals und Rand ist wahrscheinlich ähnlich der Amphore vom Pohlsberg ${ }^{2}$ ) gewesen, wenn auch wohl nicht der Rand ganz so wagerecht wie dort. Eine Parallele ist mir unbekannt; es ist meinem Urteil nach etwa Periode IV $-\mathrm{V}$ zu datieren.

Grab 12. Aus Grab 12 ist eine einhenklige Schale bekannt (Inv. 271), Höhe $10,5 \mathrm{~cm}$, Mündungsdurchmesser $29 \mathrm{~cm}$. Der Henkel ragt über den Schalenrand empor; zu jeder Seite des Henkels sitzt je eine kleine warzenförmige Erhebung; sie sind kein selbständiges Gebilde, sondern nur hochgezogene Teile des Gefässrandes. Derartige warzenförmige Erhebungen am Rande neben dem Henkel kommen bereits bei Gefässen des Bernburger Typus vor ${ }^{3}$ ).

Grab 13. Aus Grab 13 ist ein Bronzedolch und ein Gefäss bekannt. Der Bronzedolch (vielleicht Kupfer) ist ungefähr $10 \mathrm{~cm}$ lang (Inv. 320). Wahrscheinlich hat er drei Niete gehabt, von denen nur zwei noch erhalten sind.

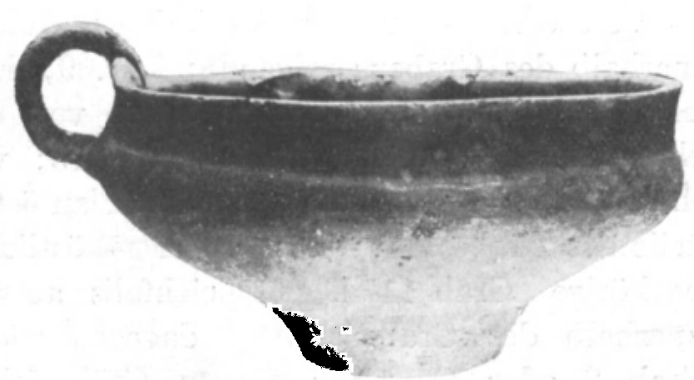

Abb. 7. Schale von Kl. Quenstedt, Windmühlenberg. $1 / 3$ nat. Gr. - Museum Halberstadt.

Eine gleiche Klinge in dem bekannten Depotfund von Pile in Schonen (Montelius, Chronologie, Abb. 158); eine andere, von Freyburg, Kr. Querfurt, stammend, ist jetzt abgebildet im Thüringer Inventarwerk, Taf. X 155 .

Das Gefäss Abb. 7 (Inv. 318) erweitert unsere Kenntnis der Keramikder ältesten Bronzezeit. Es ist eine $9,5 \mathrm{~cm}$ hohe und $19 \mathrm{~cm}$ an der Mündung messende Schale mit einem über den Rand emporragenden Henkel. Parallelen sind mir nicht bekannt. Die Zusammengehörigkeit der Funde ist vollkommen sicher.

Von den Gräbern 14--20 ist nichts bekannt. Wie auf dem von Maak herrührenden Plan des Gräberfeldes angedeutet ist, beträgt hier die Humusschicht nur $20 \mathrm{~cm}$; daher sind wohl die Gräber der Zerstörung durch den Pflug ausgesetzt gewesen. Von den Gräbern 15-20 ist ja ausdrücklich auch angegeben, dass sie bereits leer vorgefunden wurden. Über Grab 14 liess sich nichts ermitteln.

Zu besprechen bleiben noch die Gefässe Inv. 277, 316 und 317. Gefäss Inv. 316 jst eine kleine Tasse mit einem zapfenförmigen Henkel. Diese Tasse ist deshalb äusserst interessant, weil sie aus einem Aunjetitzer Becher

1) Jahresschrift VIII, Taf. I, 4 u. 5; II, 11 a.

2) Jahresschrift 1905 , S. 72 ff.

3) Stockhofhügel bei Gröna. Merkel-Höfer, Katalog des Bernburger Museums S 10. Nr. 26, S. 17 Nr 56. 
hervorgegangen ist; der Umbruch ist noch vorhanden, wenn auch nicht scharf und bereits stark in die Höhe gezogen. An dem Umbruch sitzt kein Henkel, sondern ein Zapfen, der nach Art der ansae lunatae gegabelt ist. Länge des Zapfens $1 \mathrm{~cm}$, Breite $1,5 \mathrm{~cm}$, Höhe des Gefässes 6,5 cm, Mündungsdurchmeser $9,5 \mathrm{~cm}$ :"

Gefäss Inv. 317 ist ein Aunjetitzer Becher der abgeschwächtesten Form. Der Umbruch ist ganz verschwunden; oberhalb des Henkelansatzes eine kleine Auskehlung, eine Vorstufe der Hohlkehle ${ }^{1}$ ). Höhe $6 \mathrm{~cm}$, Mündungsdurchmesser $7 \mathrm{~cm}$.

Gefäss Inv. 277 ist gleichfalls ein Aunjetitzer Becher, der oberhalb des Henkelansatzes dieselbe Auskehlung zeigt wie der vorige. Höhe $8 \mathrm{~cm}$, Mündungsdurchmesser $9,5 \mathrm{~cm}$. -

Das ganze Gräberfeld soll von einem $50 \mathrm{~cm}$ tiefen Graben umgeben gewesen sein (Abb. 1); näheres liess sich nicht feststellen.

Interessant ist die Verteilung der Gräber:

Von den sicher der Aunjetitzer Kultur angehörigen Gräbern liegen innerhalb des Grabens folgende: 1, 7, 9; hauptsächlich also im westlichen Teil. Ausserhalb des Grabens liegen von den gleichfalls dieser Kultur angehörigen Gräbern die zwei 12 und 13; von Grab 12 ist die Lage verschieden angegeben: auf dem einen Plan östlich, auf dem andern westlich an Stelle des Grabes 14. Grab 13 liegt östlich. Das der Periode IV oder V angehörige Grab 11 liegt gleichfalls ausserhalb des Grabens südöstlich. Innerhalb des Grabens liegt dagegen wieder das Grab der Latène-Zeit (Grab 2 oder 4). Aus diesem Gräberfelde werden noch Scherben der Latène-Zeit ${ }^{2}$ ) im Museum aufbewahrt, aus welchem Grabe sie aber stammen, ist nicht bekannt.

Es ist höchst bedauerlich, dass über dieses Gräberfeld keine näheren Notizen vorliegen. Wir müssen uns daher mit dem wenigen begnügen, das sich noch ermitteln liess, und so bleibt uns die Beantwortung vieler Fragen versagt. Aus diesem Gräberfelde stammen vermutlich noch viele Funde im Halberstädter Museum ${ }^{3}$ ), die nur die Bezeichnung Klein-Quenstedt tragen. Soweit sie dem Aunjetitzer Kulturkreise angehören, werde ich sie in einem später erscheinenden Aufsatz behandeln. Auch im Privatbesitz eines Magdeburger Herrn sollen sich nach 'einer Mitteilung des Herrn H. Hahne viele Aunjetitzer Funde aus der Kiesgrube befinden.

1) Erwähnen möchte ich, dass bereits ein Aunjetitzer Becher mit wirklichen Hohlkehlen bekannt, aber noch nicht veröffentlicht ist: Mus. Blankenburg a. H.

2) Wie Friederich, Beitrüge zur Altertumskunde der Grafschaft Wernigerode. $V$. 1888. Taf. II, 7.

3) Cber einen verzierten von hier stammenden Hirschhornhammer werde ich besonders berichten. 